\title{
Radiation dose to the left anterior descending coronary artery during interstitial pulsed-dose-rate brachytherapy used as a boost in breast cancer patients undergoing organ-sparing treatment
}

\author{
Marcin Sinacki, MD!, Krystyna Serkies, MD, PhD', Rafał Dziadziuszko, MD, PhD', Magdalena Narkowicz, MSc', \\ Joanna Kamińska, PhD 1,2, Joanna Lipniewicz, MScl \\ 'Department of Oncology and Radiotherapy. Medical University of Gdansk, ${ }^{2}$ Institute of Experimental Physics, Faculty of Mathematics, \\ Physics and Informatics, University of Gdansk, Poland
}

\begin{abstract}
Purpose: To assess dose received by the left anterior descending (LAD) coronary artery during interstitial pulseddose-rate brachytherapy (PDR-BT) boost for left-sided breast cancer patients undergoing organ-sparing treatment.

Material and methods: Thirty consecutive pT1-3N0-1M0 breast cancer patients boosted between 2014 and 2015 with 10 Gy/10 pulses/hour PDR-BT following a computed tomography (CT) simulation with the multi-catheter implant were included. The most common localization of primary tumor were upper quadrants. Patients were implanted with rigid tubes following breast conserving surgery and whole breast external beam irradiation (40 Gy/15 or $50 \mathrm{~Gy} / 25$ fractions). Computed tomography scans were retrospectively reviewed and LADs were contoured without and with margin of $5 \mathrm{~mm}\left(\mathrm{LAD}_{5 \mathrm{~mm}}\right)$. Standard treatment plan encompassed tumor bed determined by the surgical clips with margin of $2 \mathrm{~cm}$. Dosimetric parameters were extracted from the dose-volume histograms.

Results: The mean $\mathrm{D}_{90}$ and $\mathrm{V}_{100}$ were 10.3 Gy (range: 6.6-13.3), and 42.0 cc (range: 15.3-109.3), respectively. The median dose non-uniformity ratio (DNR) was 0.50 (range: $0.27-0.82$ ). The mean doses to $\mathrm{LAD}_{\mathrm{and}} \mathrm{LAD}_{5 \mathrm{~mm}}$ were 1.0 Gy and $0.96 \mathrm{~Gy}$, and maximal doses were $1.57 \mathrm{~Gy}$ and $1.99 \mathrm{~Gy}$, respectively. Dose to the $0.1 \mathrm{cc}$ of the LAD and $\mathrm{LAD}_{5 \mathrm{~mm}}$ were $1.42 \mathrm{~Gy}$ and $1.85 \mathrm{~Gy}$ (range: 0.01-4.98 Gy and 0.1-6.89 Gy), respectively.

Conclusions: Interstitial multi-catheter PDR-BT used as a boost for left-sided breast cancer is generally associated with low dose to the LAD. However, higher dose in individual cases may require alternative approaches.

Key words: breast carcinoma, LAD, pulsed-dose-rate brachytherapy.

\section{Purpose}

Adjuvant radiotherapy after breast-conserving surgery constitutes an indispensable part of breast conserving therapy in early breast cancer. The standard technique of radiation therapy is to treat the entire breast up to a total dose of 40-50 Gy, with or without a boost dose of 10-15 Gy to the tumor bed depending on boost indications. A decreased risk of local recurrence, particularly in younger patients, as a result of a boost dose has been confirmed in a large randomized trial [1]. For selected low-risk patients, accelerated partial breast irradiation (APBI) is an alternative treatment option to whole-breast radiotherapy (WBRT). A non-inferiority of APBI with interstitial multi-catheter brachytherapy (BT) technique was recently proved by GEC-ESTRO (Groupe Européen de Curiethérapie European Society for Radiotherapy and Oncology) trial [2]. Brachytherapy is an attractive option for both tumor-bed boost and APBI, allowing local dose escalation while reducing the dose to the surrounding normal structures.

Radiotherapy for breast cancer often involves some incidental exposure of the heart to ionizing radiation. This exposure has been associated with increased risk of cardiac morbidity and mortality [3]. Darby et al. demonstrated that the relative risk of major cardiac events including myocardial infarction, coronary revascularization, and death from ischemic heart disease, increases linearly with the mean heart dose (MHD) by $7.4 \%$ per gray with no apparent threshold [4]. The increase of the subsequent rate of ischemic heart disease in irradiated 
patients is proportional to MHD, begins within a few years after exposure, and continues for at least 20 years. Apart from individual characteristics i.e. co-morbidities and age, systemic therapy such as anthracyclines and trastuzumab may further potentiate the radiation's effect on the heart [5].

The aim of this study was to assess dose received by the left anterior descending (LAD) coronary artery during interstitial multi-catheter pulsed-dose-rate BT (PDR-BT) boost with 3-dimensional (3D) computed tomography (CT) based planning for left-sided breast cancer patients undergoing organ-sparing treatment.

Table 1. Patient and tumor characteristics $(n=30)$

\begin{tabular}{|c|c|}
\hline Parameter & $n(\%)$ \\
\hline \multicolumn{2}{|l|}{ Age (years) } \\
\hline Median (range) & $59(39-75)$ \\
\hline \multicolumn{2}{|l|}{ pT stage } \\
\hline pT1 & $22(73)$ \\
\hline pT2-3 & $8(27)$ \\
\hline \multicolumn{2}{|l|}{ pN stage } \\
\hline pNO & $25(83)$ \\
\hline $\mathrm{pN1}$ & $5(17)$ \\
\hline \multicolumn{2}{|l|}{ Histological subtype } \\
\hline Ductal & $26(87)$ \\
\hline Lobular & $3(10)$ \\
\hline Other & $1(3)$ \\
\hline \multicolumn{2}{|l|}{ Grading } \\
\hline$\overline{\mathrm{G} 1}$ & $9(30)$ \\
\hline $\mathrm{G} 2$ & $13(44)$ \\
\hline$\overline{G 3}$ & $7(23)$ \\
\hline Unknown & $1(3)$ \\
\hline \multicolumn{2}{|l|}{ WBRT } \\
\hline 40 Gy/15 fractions & $26(87)$ \\
\hline 50 Gy/25 fractions & $4(13)$ \\
\hline \multicolumn{2}{|l|}{ WBRT technique } \\
\hline 3D-tCRT & $30(100)$ \\
\hline \multicolumn{2}{|c|}{ Quadrants of the breast } \\
\hline Upper inner & $12(40)$ \\
\hline Upper outer & $10(33)$ \\
\hline Lower inner & $3(10)$ \\
\hline Lower outer & $2(7)$ \\
\hline Central & $3(10)$ \\
\hline
\end{tabular}

WBRT - whole breast radiotherapy, 3D-tCRT - 3-dimensional tangential conformal radiotherapy

\section{Material and methods}

Thirty consecutive patients were selected from women who received interstitial multi-catheter 3D PDR-BT boost dose to the conserved left breast, following WBRT at the Medical University of Gdańsk between 2014-2015 (Table 1). Rigid needles were implanted under general anesthesia. Catheter implantation was performed in a geometrically uniform way using the standardized templates for a triangular array with $16 \mathrm{~mm}$ separation of the holes. Post-implant, non-contrast CT with a slice thickness of $3 \mathrm{~mm}$ was done. The contours of PTV (planning target volume) and critical organs (skin and ribs) were imported and analyzed in treatment planning system Oncentra v. 3.3 (Nucletron, an Elekta company, Elekta AB, Stockholm, Sweden). The clinical target volume (CTV) consisted of the tumor bed with an adequate safety margin. The size of the safety margin was calculated as the sum of the width of the clear pathological surgical margins, and the radiation safety margin based on the localization of the clips left in tumor bed. It had to be approximately $20 \mathrm{~mm}$, as defined individually for every patient, considering clinical information. The PTV was contoured $5 \mathrm{~mm}$ below the skin and above the chest wall, or just above the chest wall along the pectoralis muscle for superficial and deep tumors, respectively. Paris system rules, with graphical and manual optimization to deliver adapted homogenous dose distribution to the PTV, and to keep the dose non-uniformity ratio $\left(\mathrm{V}_{150} / \mathrm{V}_{100}\right.$; $\left.\mathrm{DNR}\right)$ as close as recommended $(<0.35)$ were applied. Dose-volume histogram analyses were used to confirm that $100 \%$ of the prescribed dose covered at least $90 \%$ of the target volume. The maximum skin dose was restricted to less than $100 \%$ of the prescribed dose. A dose of 10 Gy with pulses of $1 \mathrm{~Gy} / \mathrm{h}$ was delivered.

The LAD was retrospectively contoured on the CT scans by one of the radiation oncologists, based on guidelines described by the Feng et al. [6]. Left anterior descending coronary artery was contoured from its origin to each utmost visible part on planning CT image (Figure 1). A margin of $5 \mathrm{~mm}$ was added to each LAD $\left(\mathrm{LAD}_{5 \mathrm{~mm}}\right)$ to allow for uncertainties regarding the exact position of the coronary artery. Dosimetric parameters extracted from dose-volume histograms including LAD doses of standardized plans were analyzed.

\section{Statistical analysis}

Statistical analysis was performed using SPSS software (version 20.0, IBM Corporation, USA). Quantitative data were expressed as mean \pm standard deviation (SD). For statistical analyses, four quadrants of the breast were assumed. If $>50 \%$ of the PTV was situated above the line dividing the breast into equal upper and lower portions, than the location was assigned as "upper". The same approach was applied for the medial and lateral assignments. Dose distribution to LAD, according to the tumor bed localization was compared by U-Mann Whitney test with two-sided $p$-values and significance level of $\alpha=0.05$. 

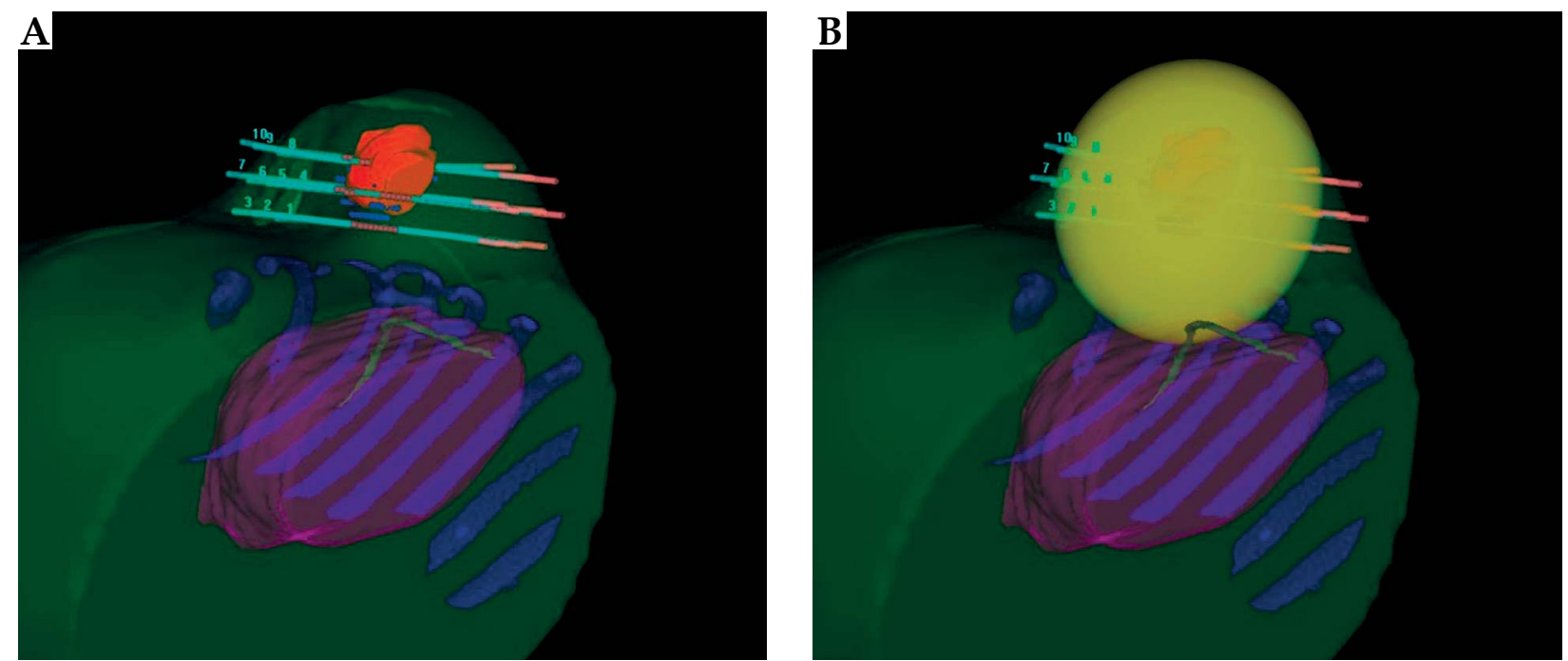

Fig. 1. Rigid needles, planning target volume, and organs at risk including left anterior descending (LAD) coronary artery (A), and isodose $10 \%$ of the prescribed dose with reference to LAD (B)

\section{Results}

Selected parameters of BT implants are presented in Table 2 . The volume enclosed by the reference isodose ranged from $15.3 \mathrm{cc}$ to $109.3 \mathrm{cc}$ (mean $42 \mathrm{cc}$ ). Tumor bed was in upper quadrants in $80 \%$ of cases, with the upper inner quadrant being the most common localization. Unsatisfactory higher DNR in half of the cases was related to our learning curve in 3D planning. A summary of radiation doses to the LAD and $\mathrm{LAD}_{5 \mathrm{~mm}}$ including $\mathrm{D}_{0.1 \mathrm{cc}}$ (representing the highest dose received by $0.1 \mathrm{cc}$ of the LAD, i.e. the dose peak) is presented in Table 3 . The average $\left(D_{\text {mean }}\right)$ and maximal dose $\left(\mathrm{D}_{\max }\right)$ to LAD were $1 \mathrm{~Gy}$ and $1.57 \mathrm{~Gy}$, respectively. There is substantial patient-to-patient variability with the LAD $D_{\max }$ up to $55 \%$ of the prescribed dose. $\mathrm{D}_{0.1 \mathrm{cc}}$ of the $\mathrm{LAD}$ and $\mathrm{LAD}_{5 \mathrm{~mm}}$ were $1.42 \mathrm{~Gy}$ and 1.85 Gy (range: 0.01-4.98 Gy and 0.1-6.89 Gy, respectively). Dose distribution to LAD according to the tumor bed localization is presented in Table 4 . There was no difference in $\mathrm{D}_{\max }$ to LAD for medial versus lateral parts of the breast $(p=0.92)$. No differences were noted for average dose according to tumor bed location (lower vs. upper quadrants; $p=0.35$, medial vs. lateral quadrants; $p=0.92$ ). $\mathrm{D}_{\max }$ to LAD was significantly higher for tumors located in lower versus upper part of the breast $(p=0.04)$.

\section{Discussion}

Micro- and macro-vascular injury in tissues within the radiotherapy field is considered the main pathophysiological cause of radiation-related heart disease [4]. The LAD artery is of particular interest by its anatomical localization within the high dose region (denoted by $95 \%$ of the radiation prescription) of the WBRT fields. An increased risk of stenosis in the LAD for left-sided radiotherapy (RT) was reported [7]. According to Darby et al., a study based on doses estimated from the RT charts, the MHD was a better predictor of the rate of major coronary events (angina episodes were not included) than the mean dose to the LAD [4].
In clinical practice, the avoidance of the heart and LAD exposure is one of the highest priorities. Several reports evaluated heart dose during breast irradiation and have provided (for standard fractionation) current recommendations of MHD and dose and volume constrains for this organ-at-risk (OAR) to keep the probability of long-term cardiac mortality $<1 \%[8,9,10,11]$. More recently, Danish

Table 2. Selected parameters of brachytherapy implants (10 Gy in 10 pulses per hour, $n=30$ )

\section{Characteristics}

\begin{tabular}{|c|c|}
\hline \multicolumn{2}{|l|}{$\mathrm{D}_{90}$} \\
\hline Median & $10.58 \mathrm{~Gy}$ \\
\hline Range & $(5.4-13.3)$ \\
\hline Percent of the prescribed dose & $54-133 \%$ \\
\hline \multicolumn{2}{|l|}{$\mathrm{V}_{100}$} \\
\hline Median & $42.03 \mathrm{cc}$ \\
\hline Range & $(15.3-109.26)$ \\
\hline \multicolumn{2}{|l|}{ DNR } \\
\hline Median & 0.5 \\
\hline Range & $(0.27-0.82)$ \\
\hline \multicolumn{2}{|l|}{ Number of tubes } \\
\hline Median & 11 \\
\hline Range & $7-15$ \\
\hline \multicolumn{2}{|l|}{ Number of implant planes } \\
\hline Two & $4(13 \%)$ \\
\hline Three & $26(87 \%)$ \\
\hline
\end{tabular}

DNR - dose non-uniformity ratio, $V_{100}$ - the percentage of the planning target volume (PTV) receiving $100 \%$ of the prescribed dose or more, $D_{90}$ - the percentage of the prescribed dose received by $90 \%$ volume of the PTV 
Table 3. Radiation dose to left anterior descending (LAD) coronary artery for 30 left-sided breast cancer patients treated with $10 \mathrm{~Gy} / 10$ pulses/hour pulsed-dose-rate brachytherapy tumor-bed boost

\begin{tabular}{lcccc} 
Dosimetric parameters & $\begin{array}{c}\text { Median dose } \\
\text { (range) }(G y)\end{array}$ & $\begin{array}{c}\text { Maximum dose } \\
\text { (range) (Gy) }\end{array}$ & $\begin{array}{c}\text { Relative to prescribed } \\
\text { dose (range) (\%) }\end{array}$ & $\begin{array}{c}\text { Mean dose } \\
\text { (range) (Gy) }\end{array}$ \\
\hline LAD & $1.4(0.1-3.9)$ & $1.57(0.1-5.5)$ & $15.7(1-55)$ & $1.0(0.1-3.4)$ \\
\hline LAD $5 \mathrm{~mm}$ & $0.8(0.1-3.0)$ & $1.99(0.1-7.5)$ & $19.9(1-75)$ & $0.96(0.1-3.0)$ \\
\hline LAD 0.1 cC & $1.33(0.01-4.98)$ & & $14.2(1.4-49.8)$ & $1.42(0.01-4.98)$ \\
\hline LAD 5 mm 0.1 cc & $1.69(0.1-6.89)$ & & $18.5(1.68-68.9)$ & $1.85(0.1-6.89)$
\end{tabular}

$L A D$ - left anterior descending coronary artery

Table 4. Dose distribution to left anterior descending (LAD) coronary artery according to the tumor bed localization

\begin{tabular}{lccc} 
Breast quadrant & Number (\%) & LAD mean dose (range) (Gy) & LAD maximal dose (range) (Gy) \\
\hline Upper outer & $10(33.3)$ & $0.83(0.1-1.5)$ & $1.19(0.1-1.8)$ \\
\hline Upper inner & $14(46.7)$ & $0.89(0.4-1.5)$ & $1.34(0.5-2.4)$ \\
\hline Lower outer & $2(6.7)$ & $1.2(1.1-1.3)$ & $2(1.7-2.4)$ \\
\hline Lower inner & $4(13.3)$ & $1.4(0.6-3.4)$ & $2.5(1-5.5)$
\end{tabular}

$\angle A D$ - left anterior descending coronary artery

experts have suggested to respect dose constrains to the heart as well as maximal dose to LAD of 20 Gy [12].

In the literature, there are limited data of LAD doses during breast irradiation, with only two published reports considering multi-catheter high-dose-rate $\mathrm{BT}$ (HDR-BT) as a form of APBI $[13,14,15,16,17,18,19,20,21]$ (Table 5). Chan et al. [14] performed a quantitative intra-patient comparison of OAR dosimetry between HDRBT versus WBRT in a group of 15 patients with left breast cancer. They contoured the whole heart to represent myocardium. The authors assumed the maximum dose for the heart as a parameter to report LAD dose. The mean LAD dose was $6.0 \mathrm{~Gy}$, which constitutes $17.6 \%$ of the prescribed BT dose of 34 Gy in 10 twice daily fractions, and $5.9 \%$ (after adjusting for $\mathrm{EQD}_{2}$ ) of $50 \mathrm{~Gy}$. Accelerated partial breast irradiation with the use of HDR-BT compared to conformal WBRT was associated with a significant reduction in radiation dose for the $\mathrm{LAD}\left(\mathrm{D}_{\text {mean }} 6.0 \mathrm{~Gy}\right.$ vs. 45.9 Gy; $p<0.01$; dose differed by a factor of 7.7). The LAD dose was the highest $(7.8 \mathrm{~Gy})$ in lower inner quadrant-located tumors. In this study, the $\mathrm{D}_{0.1 \mathrm{cc}}$ of the heart and the MHD were 16.3 Gy and 2.3 Gy for APBI, respectively, and were significantly lower than for WBRT. In Sato et al. [19] study, which compared radiation dose to the LAD during HDR-BT APBI and WBRT, LAD was outlined as in our series, but in uncertain BT cases each artery was identified with reference to the preoperative enhanced CT images to determine LAD. In the BT group (100 cases), mean PTV was 35.8 cc (6.8-135.8), mean $\mathrm{V}_{100}$ 29.8 cc (7.0-106.5), and mean DNR was 0.3 (0.2-0.6). The mean number of catheters and implant planes was 6.1 (3-12) and 1.6 (1-3), respectively. In this series, the mean and maximal LAD doses for tumors in inner quadrants or central locations $(2.54 \pm 0.21$ and $4.43 \pm 0.38 \mathrm{~Gy}$, respectively) were significantly higher than those in outer quadrants (1.02 \pm 0.17 and $2.1 \pm 0.29$ Gy, respectively; $p<0.0001)$. After adjustment of the cumulative LAD dose of APBI to WBRT using the BED equations, the mean and maximal doses in BT patients were significantly reduced in patients with tumors in outer quadrants, but increased in inner quadrants or central location. These authors stated that in patients with left-sided breast cancer, the risk of the relatively high dose to LAD should be examined before treatment. In the present series with LAD delineated according to guidelines by Feng et al., LAD dose during PDR-BT of 10 Gy boost dose applied in addition to WBRT, the $D_{\text {mean }}$ and $D_{\text {max }}$ to the LAD were on average $1 \mathrm{~Gy}$ and $1.57 \mathrm{~Gy}$, respectively. However, in some cases, the latter dose constitutes up to $55 \%$ of the prescribed dose. As the LAD is a serial organ, some authors postulated $D_{\max }$ to be applicable as a parameter in reporting LAD exposure. In our series, mean $\mathrm{D}_{0.1 \mathrm{cc}}$ was $1.42 \mathrm{~Gy}$, and ranged from 0.01 to 4.98 Gy $(0.1-49.8 \%$ of the tumor dose). We did show the difference between LAD maximal, but not average doses among patients with tumors located in the upper and lower quadrants. In a series of 60 left-sided breast cancer patients who were treated with balloon-based HDR-BT APBI using MammoSite (Hologic Inc., Bedford, MA, USA) or Contura (SenoRx, Inc., Aliso Viejo, CA, USA) applicators, for balloons located in the upper inner quadrant of the breast, the average whole heart $\mathrm{D}_{\text {mean }}$ was the highest [22]. The lowest MHD (0.51 Gy) with 3D-conformal RT (CRT) APBI as compared to multi-catheter and balloon HDR-BT APBI (1.58 Gy and $2.17 \mathrm{~Gy}$, respectively) for medially located left-breast tumors was demonstrated in an anthropomorphic phantom [23]. In the most recent study by Nilsson et al. based on coronary angiography, which was performed at a mean follow-up of 3.8 years (range: $0.3-8.2$ years) after 3D-CRT, the distribution of radiation dose in the segments of the LAD differed markedly between left- and right-sided breast cancer [17]. In the left-sided tumors in mid-LAD component and in particular segment, 8 (corresponding to distal LAD) doses approached the target of 50-60 Gy. 
Table 5. Recent studies on left anterior descending (LAD) coronary artery doses in breast cancer patients treated conservatively (mean and maximum dose - the values are averaged over analyzed patients)

\begin{tabular}{|c|c|c|c|c|}
\hline Author (Ref.) & $\begin{array}{l}\text { Number of patients/ } \\
\text { Side of the breast }\end{array}$ & $\begin{array}{c}\text { Radiotherapy, } \\
\text { type/technique/dose }\end{array}$ & $\begin{array}{c}\text { Average LAD maximum } \\
\text { dose (Gy) }\end{array}$ & $\begin{array}{l}\text { Average LAD mean dose } \\
\text { (Gy) }\end{array}$ \\
\hline \multirow[t]{3}{*}{ Bartlett et al. [13] } & \multirow[t]{3}{*}{ 34/Left-side } & WBRT 40 Gy/15 fr & & \\
\hline & & VDIBH supine & $21.0(15.8-26.2)$ & 2.9 LAD NTD $_{\text {mean }}$ \\
\hline & & FB prone & $36.8(35.2-38.4)$ & 7.8 LAD NTD mean \\
\hline \multirow[t]{2}{*}{ Chan et al. [14] } & \multirow[t]{2}{*}{ 15/Left-side } & CWBRT $50 \mathrm{~Gy} / 25 \mathrm{fr}$ & & $45.9 \pm 6.4$ \\
\hline & & HDR-BT APBI 34 Gy/10 fr & NR & $6 \pm 3.9$ \\
\hline \multirow{6}{*}{$\begin{array}{l}\text { Haclislamoglu } \\
\text { et al. [15] }\end{array}$} & \multirow[t]{6}{*}{ 10/Right-side } & WBRT 50 Gy/25 fr & & \\
\hline & & 3DCRT & $1.00 \pm 0.13$ & $0.85 \pm 0.20$ \\
\hline & & For-IMRT & $1.01 \pm 0.16$ & $0.81 \pm 0.10$ \\
\hline & & Inv-IMRT & $8.10 \pm 3.42$ & $5.05 \pm 1.56$ \\
\hline & & HT & $6.54 \pm 1.85$ & $3.49 \pm 1.20$ \\
\hline & & VMAT & $4.63 \pm 0.83$ & $3.71 \pm 0.67$ \\
\hline \multirow[t]{4}{*}{ Moorthy et al. [16] } & \multirow[t]{4}{*}{ 36/Left-side } & WBRT 45 (60) Gy/25 fr & & \\
\hline & & SIB-3DCRT gated & 39.5 Gy & NR \\
\hline & & SIB-IMRT gated & 29.17 Gy & \\
\hline & & SIB-IMRT non-gated & $35.62 \mathrm{~Gy}$ & \\
\hline \multirow[t]{2}{*}{ Nilsson et al. [17] } & 7/Left-side & \multirow[t]{2}{*}{ CRT 50-56 Gy } & $1.6-56.5^{a}$ & $1.5-55.2^{\mathrm{a}}$ \\
\hline & 8/Right-side & & $0.7-44.8^{a}$ & $0.5-21.4^{a}$ \\
\hline \multirow[t]{5}{*}{ Pham et al. [18] } & \multirow[t]{5}{*}{ 15/Left-side } & WBRT $50 / 25 \mathrm{fr}$ & & \\
\hline & & VMAT DIBH & $33.3 \pm 8.9$ & $17.4 \pm 5.8$ \\
\hline & & t-IMRT DIBH & $44.5 \pm 7.9$ & $26.0 \pm 9.5$ \\
\hline & & VMAT FB & $40.9 \pm 6.0$ & $24.7 \pm 6.5$ \\
\hline & & t-IMRT FB & $50.6 \pm 1.6$ & $39.0 \pm 6.8$ \\
\hline \multirow[t]{2}{*}{ Sato et al. [19] } & \multirow{2}{*}{$\begin{array}{l}\text { 140/Left-side (including } \\
100 \text { in APBI) }\end{array}$} & WBRT $50 / 25 \mathrm{fr}$ & $8.19 \pm 1.21(2.31-36.85)$ & $2.13 \pm 0.11(1.13-4.87)$ \\
\hline & & $\begin{array}{c}\text { HDR-BT APBI } \\
34 \text { Gy/10 fr/5-6 days }\end{array}$ & $3.15 \pm 0.26$ & $1.71 \pm 0.15$ \\
\hline \multirow[t]{2}{*}{ Taylor et al. [20] } & 50/Left-side & WBRT $40 \mathrm{~Gy} / 15 \mathrm{fr}$ & $35.2 \pm 8.8$ & $7.6 \pm 4.5$ \\
\hline & 10/Right-side & & $1.9 \pm 0.2$ & $1.6 \pm 0.2$ \\
\hline \multirow[t]{3}{*}{ Yeung et al. [21] } & 11/Left-side & WBRT 42.5 Gy/16 frx & & \\
\hline & & $\mathrm{FB}$ & NR & $10.04 \pm 8.92$ \\
\hline & & $\mathrm{DIBH}$ & & $4.20 \pm 2.77$ \\
\hline Present study & 30/Left-side & $\begin{array}{c}\text { PDR-BT boost of } 10 \\
\text { Gy/10p/h }\end{array}$ & $1.57 \pm 1.0(0.1-5.5)$ & $1.0 \pm 0.67(0.1-3.4)$ \\
\hline
\end{tabular}

WBRT - whole breast radiotherapy, VDIBH - voluntary deep-inspiratory breath-hold, FB - free-breathing, CWBRT - conformal WBRT, HDR-BT - high-dose-rate brachytherapy, $A P B I$ - partial breast irradiation, 3DCRT - three-dimensional conformal RT, IMRT - intensity modulated radiotherapy, For-IMRT - forward IMRT, InV-IMRT - inverse IMRT, HT - helical RT, VMAT - volumetric-modulated arc RT, SIB - simultaneous integrated boost, CRT - conformal RT, t-IMRT - tangential IMRT, $P D R-B T$ - pulsed-dose-rate brachytherapy

${ }^{x}$ - including 4 boosted cases, the most common WBRT dose fraction was $42.5 \mathrm{~Gy} / 16$ fraction, ${ }^{a}$ - depending on the LAD's segment

In 6 out of 7 left-breast cancer patients, it was substantially higher mean dose ranging from 30 to 55 Gy to the distal LAD than 3 to $13 \mathrm{~Gy}$ to the heart. According to these authors, mid-LAD is a critical vascular structure for developing late heart radiation effects. Patients with right-sided cancer had low MHD in a range of 1-3 Gy, and left-sided had higher MHD in a range of 3-13 Gy.
In WBRT for left-sided breast cancer, the LAD dose distribution varied among studies and depended on the technique used, being the highest with free-breathing (FB) CRT $[13,16,18,21]$. Techniques to reduce cardiac irradiation dose include two-tangential or multi-beam intensity-modulated RT (IMRT), prone positioning, and breath holding techniques. However, each of them has 
some limitations, which should be considered in clinical practice. Intensity-modulated RT in left breast cancer is associated with an improvement in dose homogeneity in the breast as well as reduction in cardiac dose, while the integral dose to the thorax, lungs, and contralateral breast is often increased. In terms of LAD dose, $\mathrm{D}_{\text {mean }}$ of the LAD for prone position is substantially lower compared to supine position, but at the expense of a higher MHD. Volumetric-modulated arc radiotherapy (VMAT) resulted in a significant further reduction in both $\mathrm{D}_{\text {mean }}$ and $\mathrm{D}_{\max }$ of the LAD compared with tangential IMRT, based on deep inspiration breath-hold (DIBH) [18]. Plans based on DIBH resulted in a dose reduction compared with FB for all heart and LAD parameters. Notably, VMAT-DIBH is associated with $30 \%$ greater $\mathrm{V}_{5 \mathrm{~Gy}}$, as well as greater dose to the contralateral breast. Study investigated supine voluntary deep-inspiratory breath-hold (VDIBH) and FB techniques to deliver $40 \mathrm{~Gy}$ in 15 fractions demonstrated significant reduction of $\mathrm{NTD}_{\text {mean }}$ of the LAD (a biologically equivalent mean of total dose to tissue normalized to 2 Gy fractions using a standard linear quadratic model, $\alpha / \beta=3$ Gy) for supine VDIBH than FB prone technique (2.9 and 7.8; $p<0.001$ ) [13]. Overall, cardiac doses were low for both techniques. Deep-inspiratory breath-hold for left-sided breast cancer was associated with $43.5 \%$ reduction in $\mathrm{D}_{\text {mean }}$ of the LAD, as compared to FB WBRT by others [21]. Comparison among current different irradiation techniques (WBRT, HDR-BT APBI, and 3D-CRT APBI) demonstrates that WBRT appears to be the most challenging, especially for patients with large breasts or when significant set-up errors are anticipated with greater probability of having cardiovascular events in the future [23]. Apart of APBI and modern external beam RT technique, intra-operative RT could further minimize dose to the coronary arteries [24].

Hypofractionated schedules of WBRT have been adopted in BCT. Most patients in our series were hypofractionated with the UK standardization of breast radiotherapy trial (START) schedule of 40 Gy in 15 fractions. Applying the Relative Seriality Model, the risk of cardiac death at 15 years of $1.4 \%$ for conventional schedule (50 Gy in 25 fractions) and $0.7 \%$ for START regimen was assumed [25]. The others reported no statistically different cardiac mortality at 15-year follow-up among left-sided breast cancer patients treated with either conventional or hypofractionated WBRT [26]. Some authors suggested that hypofractionation might be even safer in terms of heart sparing [27].

Cardiac damage is correlated to the heart-absorbed dose, which is greater for left- than right-breast irradiation and evidently in boosted patients. The mean LAD dose from left-sided WBRT was approximately five times higher than the dose from right-sided irradiation with the distal LAD receiving the highest doses (> 30 Gy; $75 \%$ of tumor dose), and the right and circumflex coronary arteries received approximately 2 Gy mean dose [20]. In this study, patient-to-patient variability in mean dose was the greatest for LAD dose from left-sided irradiation, high LAD dose was associated with high MHD, and the patient with the highest MHD (4.4 Gy) also had the high- est LAD dose (21.3 Gy). They reported that LAD $D_{\text {mean }}$ for left-tangential irradiation has decreased from $63.6 \%$ in the 1970 s to $19.0 \%$ of tumor dose in 2006. Apart from historical changes in heart doses during breast cancer $\mathrm{RT}$, the part of heart, particularly the anterior myocardial wall with LAD lying on this surface, is receiving a dose of $20 \mathrm{~Gy}$, which determine the risk of ischemic heart disease for years after RT.

A correct delineation of the heart and coronary vessels is of substantial importance. The contouring of LAD is not a routine clinical practice included in our institution. For this analysis, the LAD was retrospectively contoured by one author according to Feng et al. guidelines [6]. A multi-center study from Denmark and the UK demonstrated substantial inter-observer variation in the estimated dose to the LAD, even when guidelines for delineation were applied [12,28]. For the heart, there was little inter-observer variation in the estimated dose. Organ motion during the treatment including inter-fraction variations is another factor influencing the LAD estimated dose. In this series, the maximal dose for LAD with $5 \mathrm{~mm}$ margin was on average $1.99 \mathrm{~Gy}$, and ranged from $1 \%$ to $75 \%$ of the tumor dose. Given the geometric uncertainties, it was postulated to consider all cardiac dose parameters including $\mathrm{V}_{4 \mathrm{~Gy}}$ and $\mathrm{V}_{25 \mathrm{~Gy}}$, along with MHD, when assessing the risk of future cardiac morbidity [18]. For interstitial BT with the use of both metal and plastic catheters, the planning system applied was demonstrated to influence the dose to various volumes of interest $[29,30]$.

\section{Conclusions}

In patients with breast cancer, it is intended to minimize, as much as possible, the heart volume that is irradiated without compromising the target coverage. Recent implementation of 3D techniques has allowed delineation of the heart and coronary artery during RT planning processes including BT. The LAD dose for patients with left-sided breast cancer should be routinely examined (should be kept as low as possible) and reported to clarify its clinical consequences, and eventually establish constraints for this artery. Our data demonstrated that the mean LAD dose in interstitial multi-catheter PDR-BT boost of $10 \mathrm{~Gy}$ in-left breast is, on average, low. However, higher dose in individual cases may result in clinically relevant late cardiac toxicity; therefore, in selected patients, the boost dose may require alternative approaches.

\section{Acknowledgements}

This work was presented partially as a poster at the $5^{\text {th }}$ Congress of Polish Brachytherapy Society held in Poznan, Poland on June 2-4, 2016.

\section{Disclosure}

Authors report no conflict of interest.

\section{References}

1. Bartelink H, Maingon P, Poortmans P et al. on behalf of the European Organization for Research and Treatment of Can- 
cer Radiation Oncology and Breast Cancer Groups. Wholebreast irradiation with or without a boost for patients treated with breast-conserving surgery for early breast cancer: 20-year follow-up of a randomized phase 3 trial. Lancet Oncol 2015; 16: 47-56.

2. Strnad V, Ott OJ, Hildebrandt G et al. 5-year results of accelerated partial breast irradiation using sole interstitial multicatheter brachytherapy versus whole-breast irradiation with boost after breast-conserving surgery for low-risk invasive and in-situ carcinoma of the female breast: a randomized, phase 3, non-inferiority trial. Lancet 2016; 387: 229-238.

3. Meattini I, Guenzi M, Fozza A et al. Overview on cardiac, pulmonary and cutaneous toxicity in patients treated with adjuvant radiotherapy for breast cancer. Breast Cancer 2017; 24: 52-62.

4. Darby SC, Ewertz M, McGale P et al. Risk of ischemic heart disease in women after radiotherapy for breast cancer. N Engl J Med 2013; 368: 987-998.

5. Sardaro A, Petruzzelli MF, D'Errico MP et al. Radiation-induced cardiac damage in early left breast cancer patients: Risk factors, biological mechanisms, radiobiology, and dosimetric constrains. Radiother Oncol 2012; 103: 133-142.

6. Feng M, Moran JM, Koelling T et al. Development and validation of a heart atlas to study cardiac exposure to radiation following treatment for breast cancer. Int J Radiat Oncol Biol Phys 2011; 79: 10-18.

7. Nilsson G, Holmberg L, Garmo H et al. Distribution of coronary artery stenosis after radiation for breast cancer. J Clin Oncol 2012; 30: 380-386.

8. Gagliardi G, Constine LS, Moiseenko V et al. Radiation dose-volume effects in the heart. Int J Radiat Oncol Biol Phys 2010; 76 (3 Suppl): S77-S85.

9. Marks LB, Yorke ED, Jackson A et al. Use of normal tissue complication probability models in the clinic. Int J Radiat Oncol Biol Phys 2010; 76 (3 Suppl): S10-S19.

10. Pili G, Grimaldi L, Fidanza C et al. Geometric and dosimetric approach to determine probability of late cardiac mortality in left tangential breast irradiation: comparison between wedged beams and filed-in-field technique. Int J Radiat Oncol Biol Phys 2011; 81: 894-900.

11. Taylor CW, McGale P, Povall JM et al. Estimating cardiac exposure from breast cancer radiotherapy in clinical practice. Int J Radiat Oncol Biol Phys 2009; 73: 1061-1068.

12. Nielsen MH, Berg M, Pedersen AN et al. Delineation of target volumes and organs at risk in adjuvant radiotherapy of early breast cancer: national guidelines and contouring atlas by the Danish Breast Cancer Cooperative Group. Acta Oncol 2013; 52: 702-710.

13. Bartlett FR, Colgan RM, Donovan EM et al. The UK HeartSpare study (stage IB): randomized comparison of a voluntary breath-hold technique and prone radiotherapy after breast conserving surgery. Radiat Oncol 2015; 114: 66-72.

14. Chan TY, Tan PW, Tan CW et al. Assessing radiation exposure of the left anterior descending artery, heart and lung in patients with left breast cancer: A dosimetric comparison between multicatheter accelerated partial breast irradiation and whole breast external beam radiotherapy. Radiother Oncol 2015; 117: 459-466.

15. Haciislamoglu E, Colak F, Canyilmaz E et al. The choice of multi-beam IMRT for whole breast radiotherapy in early-stage right breast cancer. SpringerPlus 2016; 5: 688.

16. Moorthy S, Sakr H, Hasan S et al. Dosimetric study of SIBIMRT versus SIB-3DCRT for breast cancer with breath-hold gated technique. Int J Cancer Ther Oncol 2014; 2: 1-9.

17. Nilsson G, Witt Nyström GN, Isacsson U et al. Radiation dose distribution in coronary arteries in breast cancer radiotherapy. Acta Oncol 2016; 55: 959-963.
18. Pham TT, Ward R, Latty D et al. Left-sided breast cancer loco-regional radiotherapy with deep inspiration breath-hold: Does volumetric-modulated arc radiotherapy reduce heart dose further compared with tangential intensity-modulated radiotherapy? J Med Imaging Radiat Oncol 2016; 60: 545-553.

19. Sato K, Mizuno Y, Fuchikami H et al. Comparison of radiation dose to the left anterior descending artery by whole and partial breast irradiation in breast cancer patients. J Contemp Brachytherapy 2015; 7: 23-28.

20. Taylor CW, Povall JM, McGale P et al. Cardiac dose from tangential breast cancer radiotherapy in the year 2006. Int J Radiat Oncol Biol Phys 2008; 72: 501-507.

21. Yeung R, Conroy L, Long $\mathrm{K}$ et al. Cardiac dose reduction with deep inspiration breath hold for left-sided breast cancer radiotherapy patients with and without regional nodal irradiation. Radiat Oncol 2015; 10: 200.

22. Valakh V, Kim Y, Werts ED et al. A comprehensive analysis of cardiac dose in balloon-based high-dose-rate brachytherapy for left-sided breast cancer. Int J Radiat Oncol Biol Phys 2012; 82: 1698-1705.

23. Merino Lara TR, Fleury E, Mashouf S et al. Measurement of mean cardiac dose for various breast irradiation techniques and corresponding risk of major cardiovascular event. Front Oncol 2014; 4: 284.

24. Pezner RD. Coronary artery disease and breast radiation therapy. Int J Radiat Biol Phys 2013; 86: 816-818.

25. Portaluri M, Grimaldi L, D'Errico MP et al. Hypofractionated radiotherapy of the breast may be safer for the heart. Breast 2011; 20: 481-484.

26. Chan EK, Woods R, Virani S et al. Long-term mortality from cardiac causes after adjuvant hypofractionated vs. conventional radiotherapy for localized left-sided breast cancer. Radiother Oncol 2014; 113: 73-78.

27. Appelt AL, Vogelius IR, Bentzen SM et al. Modern hypofractionation schedules for tangential whole breast irradiation decrease the fraction size-corrected dose to the heart. Clin Oncol (R Coll Radiol) 2013; 25: 147-152.

28. Lorenzen EL, Taylor CW, Marando $M$ et al. Inter-observer variation in delineation of the heart and left anterior descending coronary artery in radiotherapy for breast cancer: A multi-centre study from Denmark and the UK. Radiother Oncol 2013; 108: 254-258.

29. Hofbauer J, Kirisits Ch, Resch A et al. Impact of heterogeneity-corrected dose calculation using a grid-based Boltzmann solver on breast and cervix cancer brachytherapy. J Contemp Brachytherapy 2016; 8: 143-149.

30. Sinnatamby M, Nagarajan V, Reddy S et al. Dosimetric comparison of Acuros BV with AAPM TH43 dose calculation formalism in breast interstitial high-dose-rate brachytherapy with the use of metal catheters. J Contemp Brachytherapy 2015; 7: 273-279. 